\title{
Half-metallic finite zigzag single-walled carbon nanotubes from first principles
}

\author{
A. Mañanes* and F. Duque \\ Departamento de Física Moderna, Universidad de Cantabria, E-39005 Santander, Spain
}

\author{
A. Ayuela \\ Centro de Física de Materiales (CFM), CSIC-Universidad del País Vasco, E-20018 San Sebastián, Spain \\ and Donostia International Physics Center (DIPC), E-20018 San Sebastián, Spain \\ M. J. López and J. A. Alonso ${ }^{\dagger}$ \\ Departamento de Física Teórica, Atómica y Óptica, Universidad de Valladolid, E-47005 Valladolid, Spain
}

(Received 7 March 2008; revised manuscript received 30 May 2008; published 17 July 2008)

\begin{abstract}
Density-functional calculations predict half-metallicity in zigzag single-walled carbon nanotubes of finite length with the two ends saturated with hydrogen. We have analyzed the change of the $\alpha$-and $\beta$-spin electronic gaps under the influence of an electric field applied along the nanotube axis. The half-metallic behavior, in which the electronic gap is zero for one spin flavor and nonzero for the other, is obtained for a critical electric field of 3.0/w V/ $\AA$, where $w$ is the length of the nanotube. This critical field is the same as that predicted for graphene nanoribbons. By a detailed analysis of the spin structure of the ground state, we show the relevance of the edge states, electronic states spatially localized at the carbon atoms of the nanotube boundaries, on the on-set of half-metallicity, and on the magnetic properties of the finite semiconducting zigzag nanotubes.
\end{abstract}

DOI: 10.1103/PhysRevB.78.035432

PACS number(s): 73.22.-f, 75.75.+a, 72.80.Rj, 85.75.-d

\section{INTRODUCTION}

Recent calculations based on density-functional theory (DFT) indicate that zigzag graphene nanoribbons, a single graphite layer of finite width and infinite length terminated by hydrogen-passivated zigzag edges on both lateral sides (ZGNR), present half-metallicity in the presence of a coplanar electric field perpendicular to the zigzag boundaries. ${ }^{1,2}$ The effect is also present considering different oxidation schemes at the edges of the zigzag nanoribbon, for example, full hydroxylation (OH groups), ${ }^{2}$ and also in finite rectangular graphene nanoribbons. ${ }^{3}$ In spite of some controversy, ${ }^{4}$ the study of finite graphene structures by Hod et al. ${ }^{3}$ has established that the half-metallic nature of the zigzag ribbons (whether periodic or finite) is robust and insensitive to the level of theory used. Idealized half-metals have only one spin channel for conduction: the spin-polarized density of states exhibits metallic behavior for one spin direction, while for the other spin channel there is a gap at the Fermi level. ${ }^{5,6}$ It has been stressed ${ }^{1}$ that the interplay found between electric fields and the electronic spin degree of freedom opens a new path to develop spintronics at the nanometer scale using carbon-based devices. ${ }^{7}$

Half-metallicity induced in the ZGNRs by the electric field is related to the existence of electronic states at the Fermi energy spatially localized at the zigzag edges. ${ }^{1}$ The ground-state spin configuration at zero electric field corresponds to an opposite spin orientation across the ribbon [antiferromagnetic (AFM)] between the spin-polarized edge states. That is, the spins on the outermost sites of each zigzag edge are parallel, resulting in one edge polarized with spin up and the other polarized with spin down, and the total ZGNR spin is equal to zero. The ferromagnetic (FM) state, with the spins at the two zigzag edges aligned in the same direction, results in a metastable phase, and following this one there is a nonmagnetic phase (NM), as have been found in $a b$ initio calculations. ${ }^{8}$ Calculations for zigzag-edged carbon strips without hydrogen termination indicate that the AFM state is also the lowest-energy state. ${ }^{9}$ In contrast, the armchair-edged nanoribbons do not show such magnetic phases due to the delocalized nature of the frontier orbitals. ${ }^{10}$ Similar magnetic effects related to the electronic states spatially localized at the zigzag edges have been also predicted for finite graphene nanoislands. ${ }^{3,411-14}$ The presence of edge states in graphitic systems has been confirmed by scanning tunneling microscopy and spectroscopy, which show a clear peak in the local density of states near the zigzag edges, while such a peak was not observed near the armchair edges. ${ }^{15,16}$

The interest of carbon nanotubes in nanotechnology has motivated a large number of studies. ${ }^{17,18}$ Realistic electronic devices can be produced only using finite-size single-wall nanotubes (SWNTs), which are either connected to metal leads or lie on a substrate, and this has motivated the study of the electronic properties of nanotubes of finite length. A relevant result is that finite zigzag nanotubes also have localized states at the tube edges but not the armchair tubes. ${ }^{10}$ First-principles calculations of the magnetic properties of finite zigzag SWNTs have been performed as a function of their radius and length, ${ }^{19,20}$ and those calculations have shown the importance of the localized edge states on the magnetism of the finite nanotubes. Further spin-polarized DFT calculations of the magnetic properties of carbon nanostructures have shown not only the relevance of the edge states located at the undercoordinated carbon atoms of the zigzag boundaries ${ }^{21,22}$ but also the influence of stabilized radicals formed by trivalent carbons, introduced within the aromatic system of the otherwise $s p^{2}$-bonded tetravalent carbon atoms in producing unpaired spins in the electronic ground state. ${ }^{22}$

The results of the present work indicate that finite pieces of zigzag single-walled carbon nanotubes behave as half- 
metallic systems under the influence of an electric field. Using DFT we have studied finite pieces of a zigzag $(14,0)$ SWNT, focusing attention on the evolution of the electronic gap of the two spin flavors as a function of the intensity of an external electric field applied along the axis of the nanotube. As in the case of ZGNRs, the onset of half-metallicity is related to the existence of electronic states at the Fermi energy localized at each edge of the nanotube, which in the absence of the applied field are degenerate. Due to the spatial localization of these states with energies around the Fermi level, the effects of the external axial fields on them are significant, in contrast with the extended states. We have not found the effect of half-metallicity in the case of finite nanotubes derived from the $(8,8)$ armchair SWNT because in this case the frontier orbitals are spatially delocalized over the finite nanotube and have small amplitudes at the edges.

In Sec. II we briefly explain the method used to solve the Kohn-Sham equations of DFT and we describe the two finite pieces of the $(14,0)$ nanotube that we have analyzed, $\mathrm{C}_{168} \mathrm{H}_{28}$ and $\mathrm{C}_{112} \mathrm{H}_{28}$. In Sec. III A we first calculate the binding energies obtained for different magnetic configurations of the free nanotube and of the nanotube under an electric field $E$ applied along the nanotube axis. In all cases the ground state is the singlet $S=0$ AFM state. The values of the parallel static dipole polarizability are calculated from the linear dependence of the binding energy on the square of the electric field $E^{2}$. In Sec. III B we study the properties of the electronic density of states both for the AFM ground state and for the NM state. For the AFM state, one side of the nanotube is dominated by $\alpha$-spin states and the other by $\beta$-spin states coupled to a total spin of zero. In Sec. III C we present the variation of the electronic spin gaps when an electric field is applied along the axis of the nanotube. The behavior of the gaps is similar for the two finite nanotubes considered. The main result is that the critical field required to establish halfmetallicity is the same for both tubes, 3.0/w (V/ $\AA$ ), where $w$ is the length of the nanotube. This critical field is equal to the one obtained for ZGNRs. At this critical field the gap for one of the spin channels is zero and that for the other is a maximum. We also analyze the behavior, under the influence of the electric field, of the electronic states near the Fermi level. For large values of the electric field we reach the threshold for field emission. In Sec. IV we present the conclusions of this work.

\section{METHOD}

Our DFT calculations use the ADF 2006.01 code $^{23,24}$ with the local-spin-density approximation (LSDA) of Vosko et $a l{ }^{25}$ for the exchange-correlation energy. The frozen-core approximation is used for the helium core of the carbon atoms and four valence electrons are considered for each carbon. The basis set is formed by localized atomic orbitals: a triple- $\zeta$ basis with one polarization function, formed by four $s$, three $p$, and one $d$ Slater-type atomic orbitals for each carbon atom, and three $s$ and one $p$ orbitals for each hydrogen atom. The code makes full use of any molecular symmetry present in the system using orthogonalized symmetryadapted molecular orbitals to solve the Kohn-Sham equations, and the binding energy is calculated with respect to atomic fragments. The atomic positions are optimized using a conjugate gradient method where the binding energy and the atomic forces are minimized. For spin-unrestricted calculations, like in the present case, it is possible to tune the initial potential by allowing different spin configurations even for equivalent atoms, thus allowing for spin brokensymmetry calculations. Furthermore, the code allows for the application of a constant electric field along a specific direction.

We have considered two finite pieces of the zigzag $(14,0)$ SWNT using hydrogen atoms to saturate the dangling bonds of the carbon atoms at the edges. In the first case, we consider the aromatic molecule $\mathrm{C}_{168} \mathrm{H}_{28}$ for which a complete relaxation of the carbon-carbon and carbon-hydrogen distances has been performed within $D_{7}$ symmetry for the structure. This finite nanotube has six zigzag rings, each one with 28 carbon atoms, the minimum carbon-carbon distance is $d_{\mathrm{CC}}=1.41 \AA$, the nanotube radius is $R=5.45 \AA$, and the length along the axis of the tube is $w_{1}=8 d_{\mathrm{CC}}=11.28 \AA$. There are also two external rings, of 14 hydrogen atoms each, located at distance $d_{\mathrm{CH}}=1.105 \AA$ from the most external carbon atoms, to saturate the bonds. In the second case, we consider a shorter piece of the zigzag $(14,0)$ SWNT, $\mathrm{C}_{112} \mathrm{H}_{28}$ with only four zigzag rings but with the same number of hydrogen atoms at the boundaries. After a new relaxation of the geometry, again within the $\mathrm{D}_{7}$ symmetry, the length results $w_{2}=5 d_{\mathrm{CC}}=7.05 \AA$ but with the same radius as the previous fragment. In this shorter nanotube, the relaxation of the geometry induces only small distortions of the $d_{\mathrm{CC}}$ distances with respect to those of the longer tube, only for those carbon atoms at the boundaries of the finite nanotube.

\section{RESULTS}

\section{A. Binding energy and interaction energy with the external electric field}

In this section we focus mainly on the finite $\mathrm{C}_{168} \mathrm{H}_{28}$ nanotube because analogous results have been obtained for the shorter nanotube. First, the initial $D_{7}$ symmetry of the structure is reduced to $\mathrm{C}_{7 V}$ to allow for different spin orientations at the two ends of the SWNT. When the electric field is zero, we found that the configuration with opposite spin orientations at the two edges of the nanotube, that is, the AFM state in which the highest occupied molecular orbital (HOMO) of one spin orientation HOMO- $\alpha$ is an edge state localized on one end of the tube and the HOMO- $\beta$ is localized on the opposite side, is favored over a configuration in which both HOMO- $\alpha$ and HOMO- $\beta$ have equal probabilities on both sides of the nanotube (the NM sate). In Fig. 1 we present the calculated binding energies as

$$
E_{b}\left(\mathrm{C}_{168} \mathrm{H}_{28} ; S, E\right)=E_{T}\left(\mathrm{C}_{168} \mathrm{H}_{28} ; S, E\right)-168 E_{T}(\mathrm{C})-28 E_{T}(\mathrm{H}) .
$$

For each value of the electric field $E$ and of the spin $S$ $=\left(n_{\alpha}-n_{\beta}\right) / 2$, where $n_{\alpha}$ and $n_{\beta}$ are the number of electrons with up and down spins, respectively, the binding energies $E_{b}$ 


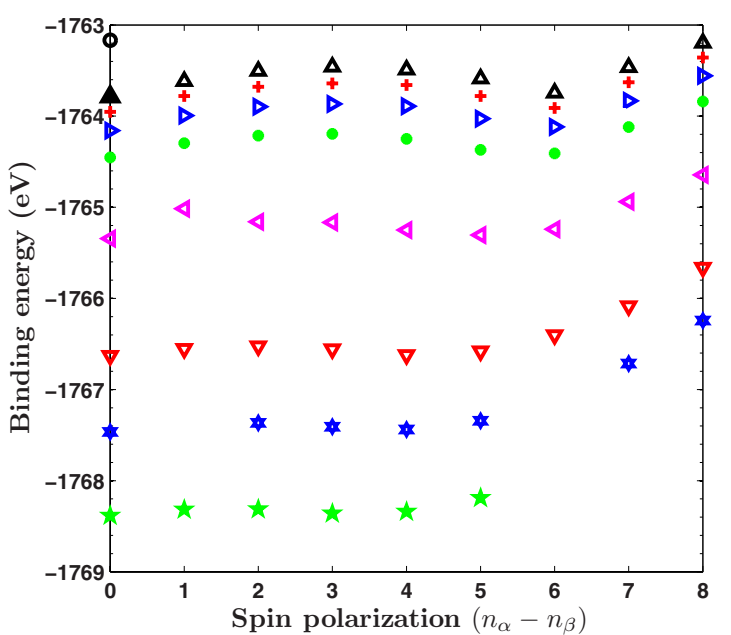

FIG. 1. (Color online) Binding energy for the finite zigzag $(14,0)$ SWNT $\mathrm{C}_{168} \mathrm{H}_{28}$ as a function of the spin polarization $\left(n_{\alpha}\right.$ $-n_{\beta}$ ) for different values of the electric field $E$ parallel to the tube axis. Different symbols correspond to different values of the electric field (in $\mathrm{V} / \AA$ ): black vertical triangles $(E=0)$, red crosses $(E$ $=0.10)$, blue triangles pointing to the right side $(E=0.15)$, green dots $(E=0.21)$, magenta triangles pointing to the left $(E=0.31)$, red triangles pointing down $(E=0.41)$, blue stars $(E=0.46)$, and green pentagonal stars $(E=0.51)$. For all values of the electric field the ground state is $\left(n_{\alpha}-n_{\beta}\right)=0$, the AFM state. The full black triangle at $\left(n_{\alpha}-n_{\beta}\right)=0$ is the AFM ground state for $E=0$, and the empty circle gives the binding energy for the nonmagnetic state at $E=0$.

are the atomization energies calculated from the total energies $E_{T}$. The binding energies at $E=0$ of these two states, AFM and NM, with zero total spin $(S=0)$, are indicated in Fig. 1 by a black filled vertical triangle and by a circle, respectively, at the left, which is for $n_{\alpha}-n_{\beta}=0$. Moreover, the $S=0$ AFM state results the true ground state even when the spontaneous magnetization of the finite nanotube is allowed, as is indicated by the set of empty black vertical triangles in Fig. 1. A local minimum is found for $n_{\alpha}-n_{\beta}=6$, so $S=3$. This local minimum, less stable than the ground state by $45 \mathrm{meV}$, corresponds to a FM state.

The calculated magnetic interaction energies are large, as in the case of ZGNRs. ${ }^{1}$ For example, the difference in binding energies between the FM state with spin-polarized edges (the local minimum with $S=3$ ) and the unpolarized NM state (the empty circle at $S=0$ in Fig. 1) is $577 \mathrm{meV}$, which gives $20.6 \mathrm{meV}$ per carbon atom at the edge (there are 28 edge atoms), in complete agreement with the $20 \mathrm{meV}$ per edge atom calculated for ZGNRs. ${ }^{1}$ Furthermore, when the antiferromagnetic coupling between the spin-polarized edges is allowed, then the ground state represented by the filled black triangle in Fig. 1 is obtained, and the stability is further increased by $45 \mathrm{meV}$, that is, $1.6 \mathrm{meV}$ per edge atom, again in agreement with the increase of $2 \mathrm{meV}$ per edge atom found for a ZGNR formed by eight zigzag chains. ${ }^{1}$ In our case the number of zigzag carbon chains in the system is 6 . This FM-AFM exchange coupling energy per border $\mathrm{C}$ atom of $1.6 \mathrm{meV}$ can be compared with typical values calculated for multilayers of magnetic elements. For example, the exchange coupling energy of Co or Fe layers sandwiched between metallic $\mathrm{Cu}$ spacer layers is $2 \mathrm{meV}$ per atom, although it be- comes 1 order of magnitude smaller for increasing spacer distances. ${ }^{26} \mathrm{In} \mathrm{Co} / \mathrm{Si}$ multilayers, where the spacer is a semiconductor, the exchange energy is $4 \mathrm{meV}$ per atom for $4-6$ Si monolayers. ${ }^{27}$

For the two nanotubes considered, with 168 and 112 carbon atoms, respectively, the calculated energy differences between the NM state and the FM state (in both cases the FM state is a local minimum with $S=3$ ) are, respectively, 3.43 and $4.3 \mathrm{meV}$ per carbon atom. These values are of the same order of magnitude as the corresponding ones for nanostructures of typical magnetic transition metals. For instance, they are comparable with the calculated value of $7.7 \mathrm{meV}$ per atom obtained for fcc magnetic iron clusters $\mathrm{Fe}_{n}$ with $n$ $=13-19 .{ }^{28}$ In spite of the fact that the actual ground states of the considered nanotubes are the AFM states with $S=0$ (which are only 45 and $50 \mathrm{meV}$ below the FM state), our results support the possibility of implementing stable nanomagnets using finite pieces of zigzag carbon nanotubes. ${ }^{19,20}$

When an axially directed electric field of intensity $E$ is applied, the binding energies behave in a similar way as for zero electric field. The results are displayed also in Fig. 1. For all values of the electric field considered, the absolute energy minimum corresponds to the AFM state with $S=0$, and a second minimum, less than $45 \mathrm{meV}$ above the main one, is found for a value of the spin polarization that decreases as the electric field is increased: from $S=3$ for $E=0$ to $S=3 / 2$ for $E=0.51 \mathrm{~V} / \AA$. The energy difference, per boundary carbon atom, between the FM states and the ground state (AFM) calculated as

$$
\Delta E_{m}=\left\{E_{b}(S, E)-E_{b}(S=0, E)\right\} / 28,
$$

is shown in Fig. 2 as a function of the spin polarization $\left(n_{\alpha}-n_{\beta}\right)$ for different values of the intensity of the electric field. The upper panel corresponds to the long nanotube and the lower panel to the short nanotube.

To clarify the figure, the energy zero has been shifted by a different amount for each electric field, as explained in Fig. 2. For both nanotubes and for $E$ smaller than $0.30 \mathrm{~V} / \AA$, there is a metastable FM state at $S=3$. The maximum energy difference between this FM state and the ground state is 45 $\mathrm{meV}(50 \mathrm{meV})$, where the value for the short nanotube is given in parentheses. This amounts to an energy difference of $1.6 \mathrm{meV}(1.8 \mathrm{meV})$ per edge carbon atom. These values are in full agreement with recent results by Hod et al. ${ }^{3}$ for finite nanoribbons with $2 \times 14$ zigzag edge carbon atoms: using LSDA they obtain values between 60 and $70 \mathrm{meV}$ for the difference between the FM and the AFM states at zero electric field. Hod et $a l^{3}{ }^{3}$ showed also that the calculated energy differences increase when nonlocal or hybrid functionals are used for the exchange-correlation energy.

The results in Fig. 2 for higher values of the electric field indicate that the FM minimum is found in the region between $S=5 / 2$ and $S=3 / 2$, and that the energy difference is smaller for both tubes than in the case of $E=0$. We stress that for $n_{\alpha}-n_{\beta}=0$ and $E \neq 0$, the electronic structure relaxes to the AFM state in the cases analyzed even if the calculation starts from the NM spin structure. From the small differences between the AFM and FM energies shown in Fig. 2 one could think that changes from AFM to FM states could occur 

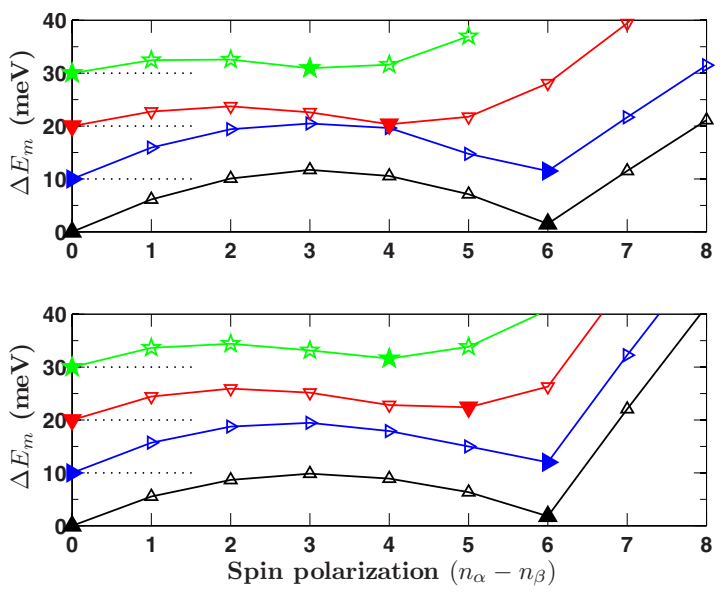

FIG. 2. (Color online) Magnetic energy per edge carbon atom, calculated using Eq. (2) as the energy difference between the FM state with spin $S=\left(n_{\alpha}-n_{\beta}\right) / 2$ and the ground state, AFM, with $S$ $=0$ as a function of the spin polarization for several values of the electric field $E$ (in $\mathrm{V} / \AA$ ) : black vertical triangle, $E=0$; blue triangle pointing to the right side, $E=0.15$; red triangle pointing down, $E$ $=0.41$; and star, $E=0.51$. The upper panel corresponds to the nanotube $\mathrm{C}_{168} \mathrm{H}_{28}$ and the lower one to the $\mathrm{C}_{112} \mathrm{H}_{28}$ nanotube. Except for $E=0$, the energies have been shifted so that the horizontal dashed line corresponds to zero energy difference. The full symbols indicate the location of the two minima: the absolute one at $S=0$ and the metastable state at $S>0$.

easily by thermal fluctuations. However, a closer analysis of Fig. 2 shows that the total-energy barriers between these two states, obtained from Eq. (2) as $28 \times \Delta E_{m}$, where $\Delta E_{m}$ is now the difference in binding energy between the AFM minimum on the left of each curve and the maximum in the middle of the same curve, are quite large for low electric fields, 330 $\mathrm{meV}(275 \mathrm{meV})$ for $E=0$, thus preventing the thermal gradual switching between AFM and FM states. When the electric field is increased the energy barrier decreases, but even for the more intense field in Fig. $2, E=0.51 \mathrm{~V} / \AA$, the barriers are still substantial: $100 \mathrm{meV}(120 \mathrm{meV})$. These values of the barrier heights can be considered as upper bounds of the true barriers, as far as we have only considered collinear spin configurations, and there may be noncollinear spin arrangements that can serve as transition states between the AFM ground state and the FM spin state. The trend observed in Fig. 2 indicates that only the AFM minimum with $S=0$ will survive at higher values of the electric field. However, for strong electric fields, electrostrictive effects should be taken into account, as will be discussed in Sec. III. In any case, the magnetic effects discussed here are associated to localized edge states that we study in Sec. III B. These effects show up due to the substantial proportion of atoms at the zigzag edges.

The variation of the binding energy of the AFM ground state, $E_{b}(E)$ of Eq. (1), with the applied electric field, is given in Fig. 3. According to perturbation theory, ${ }^{29}$ the binding energy should present a quadratic dependence on an electric field applied along the axis of the tube because of the $\pm z$ symmetry of the unperturbed electronic charge, so $E_{b}(E)$ $=E_{b}(0)-(1 / 2) \alpha_{\|} E^{2}$. The predicted quadratic behavior is obtained in our calculations, as can be seen in Fig. 3. The value

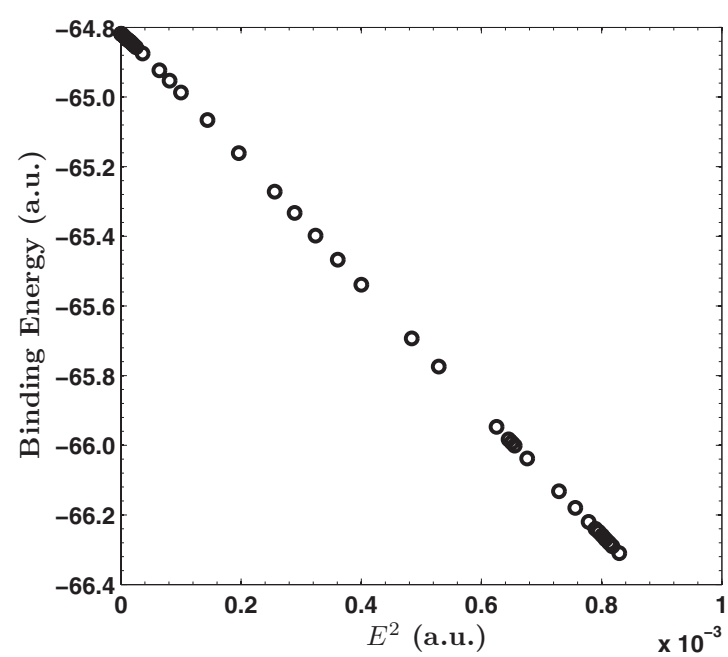

FIG. 3. Binding energy (in atomic units) of the AFM state for a zigzag $(14,0) \mathrm{C}_{168} \mathrm{H}_{28}$ SWNT as a function of the square of the electric field, $E^{2}$. The electric field is applied along the axis of the nanotube ( 1 a.u. of electric field is equal to $51.42 \mathrm{~V} / \AA$ ).

obtained for the longitudinal static screened dipole polarizability (which is the experimentally accessible quantity) of the longer nanotube is $\alpha_{\|}\left(w_{1}\right)=563.3 \AA^{3}$. The polarizability per unit length is $\left(1 / w_{1}\right) \alpha_{\|}\left(w_{1}\right)=47.5 \AA^{2}$. For the shorter tube, the behavior of $E_{b}(E)$ is similar to that shown in Fig. 3 but with a lower value of the longitudinal polarizability, $\alpha_{\|}\left(w_{2}\right)=274.6 \AA^{3}$, which gives $\left(1 / w_{2}\right) \alpha_{\|}\left(w_{2}\right)=38.95 \AA^{2}$. These values for the polarizability per unit length are smaller than the calculated value ${ }^{30}$ for an infinite $(14,0)$ nanotube: $\alpha_{z z}=268.3 \AA^{2}$. In a nanotube of infinite length the longitudinal screened and unscreened polarizabilities are identical because polarization charges are not induced for a field along the tube axis. The lower values obtained for finite nanotubes are associated to a finite-size effect because in this case polarization charges are induced on the extremes of the nanotube, thus producing a reduction in the total electric field felt by the electrons, and consequently a reduction in the screened (experimental) polarizability with respect to the unscreened value. Furthermore, we have obtained $\alpha_{\|}\left(w_{2}\right) / w_{2}$ $<\alpha_{\|}\left(w_{1}\right) / w_{1}$ for $w_{2}<w_{1}$, in agreement with the trends of the finite-size corrections proposed in Ref. 30. For the two finite nanotubes considered, the polarizabilities per carbon atom, $\alpha_{0}\left(w_{1}\right)=3.2$ and $\alpha_{0}\left(w_{2}\right)=2.45 \AA^{3}$, are of the same order as for the $\mathrm{C}_{60}$ fullerene, $1.38-1.42 \AA^{3}$ per atom. ${ }^{31}$

\section{B. Characterization of the edge states}

The calculated electronic densities of states (DOS) of the $\mathrm{C}_{168} \mathrm{H}_{28}$ nanotube in different magnetic states are shown in Figs. 4-7. Figure 4 gives the DOS for the NM state in the absence of electric field; this state corresponds to the circle on the left axis of Fig. 1. This $S=0$ state is obtained in a constrained calculation with the restrictions of $D_{7}$ symmetry and equal electronic spin densities, $\rho_{\alpha}(\vec{r})=\rho_{\beta}(\vec{r})$. There are several near-degenerate states at the Fermi level in an energy interval of $0.02 \mathrm{eV}$ : one $A_{1}$ and two $E_{1}$ occupied states and two $E_{1}$ and one $A_{1}$ unoccupied states. These states are the 

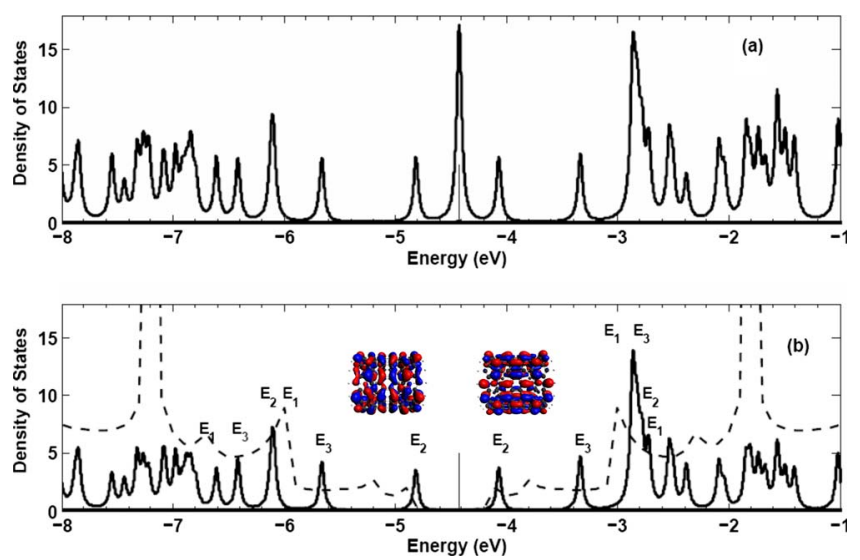

FIG. 4. (Color online) Calculated electronic DOS (arbitrary units) for a finite zigzag $(14,0)$ SWNT $\mathrm{C}_{168} \mathrm{H}_{28}$ in the absence of electric field. The DOS corresponds to a constrained calculation for a NM state with $D_{7}$ symmetry (the circle in Fig. 1). The total DOS is given in panel (a), and the position of the near degenerated HOMO and LUMO is indicated by a vertical line. The DOS obtained by excluding the edge states, those located over the two more external carbon rings, is given in panel (b). The insets give the wave functions of the $E_{2}$ states below the HOMO (left) and above the LUMO (right). The dashed curve gives the DOS calculated in a $\pi$-tight-binding model for an infinite nanotube (Ref. 32). The labels in (b) correspond to the $D_{7}$ symmetry of the system. A Lorentzian of width $0.05 \mathrm{eV}$ has been used to broaden each eigenvalue.

edge states shown in the enlarged view given in Fig. 5, where we present also the spatial distributions of the wave functions of these states. These edge states are nearly degenerate in energy and they are localized on the most external carbon rings of the finite nanotube. When the contributions to the DOS coming from the carbon atoms at the two more

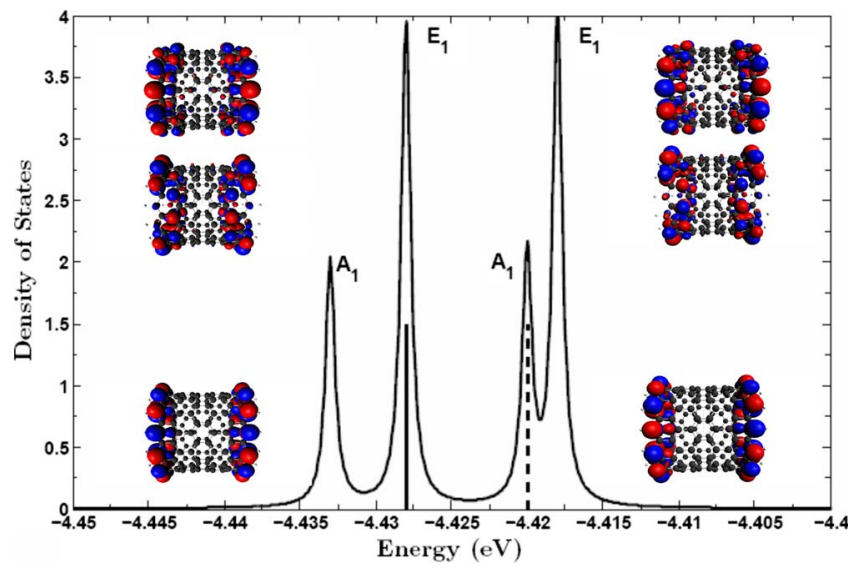

FIG. 5. (Color online) Expanded view of the DOS (arbitrary units) of the NM state of Fig. 4(a) in the region near the Fermi level. The HOMO $\left(E_{1}\right)$ is indicated by a vertical line and the LUMO $\left(A_{1}\right)$ by a vertical dashed line. The insets correspond to the wave functions of (from up to bottom and from left to right): the twofold degenerated $E_{1}$ HOMO, the occupied $A_{1}$, the twofold degenerated unoccupied $E_{1}$ states, and the LUMO $A_{1}$. Red and blue indicate positive and negative values of the wave function. A Lorentzian of width of $0.00075 \mathrm{eV}$ has been used to broaden each eigenvalue.
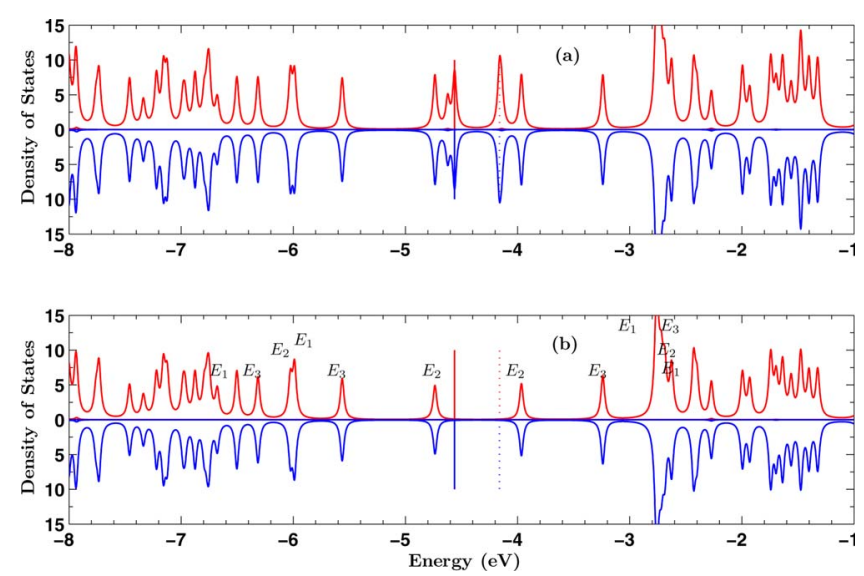

FIG. 6. (Color online) Calculated spin $\alpha$ (red) and $\beta$ (blue) electronic DOS (arbitrary units) for the $S=0$ AFM state in the absence of electric field. The symmetry used is $C_{7 V}$. (a) Total DOS of the finite nanotube with the HOMO and LUMO energies indicated by a vertical line and a vertical dashed line, respectively. Compared to Figs. 4(a) and 5, the inclusion of the spin degree of freedom breaks the near degeneracy HOMO-LUMO. In (b), the edge states have been excluded.

external rings (14 carbon atoms on each ring) are excluded, we obtain the DOS of Fig. 4(b), where we notice that not only the HOMO and LUMO but also the whole group of states very close to the Fermi level have disappeared. The DOS in Fig. 4(b) can be considered as the DOS of the central region of the nanotube, where border effects have been excluded. The two insets in Fig. 4(b) show the wave functions for the two $E_{2}$ states closer to the Fermi level, and they indicate that these states have their densities distributed along the whole finite nanotube. The DOS of an infinite $(14,0)$ tube calculated from a tight-binding (TB) model for the $\pi$ electrons which includes curvature effects ${ }^{32}$ is also given as a dashed curve in Fig. 4(b). From the comparison of

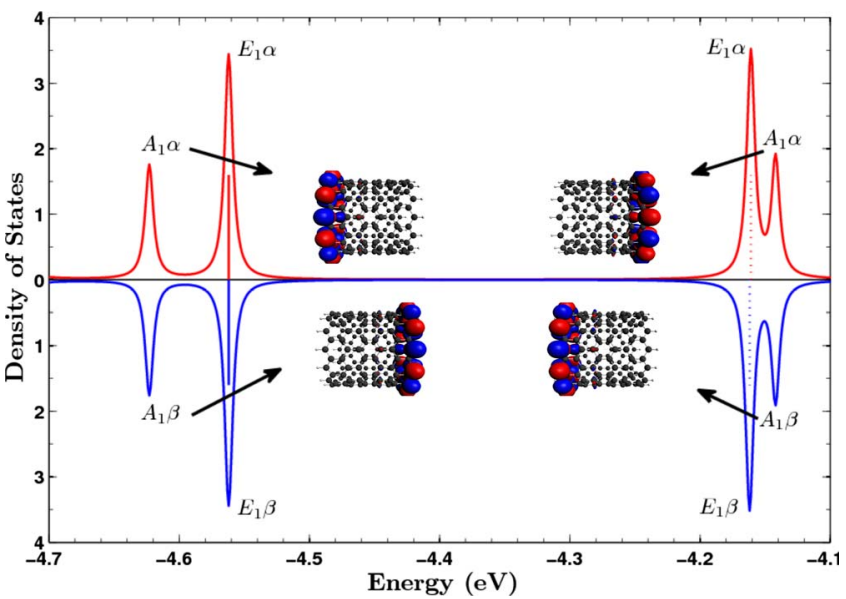

FIG. 7. (Color online) Expanded view of the DOS (arbitrary units) in the region of the $S=0$ AFM state of Fig. 6(a) near the HOMO and LUMO. The present DOS corresponds to that part excluded in Fig. 6(b). The insets give the wave functions corresponding to the indicated $\alpha$ and $\beta A_{1}$ states. A Lorentzian of width of $0.0075 \mathrm{eV}$ has been used to broaden each eigenvalue. 


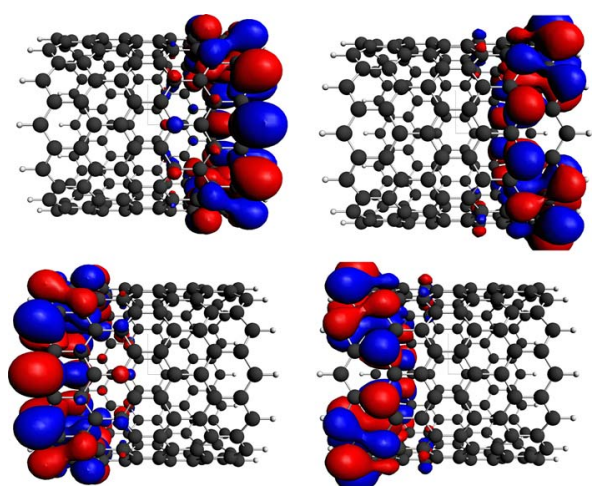

FIG. 8. (Color online) $\alpha$-spin LUMO and HOMO orbitals of the AFM ground state. The upper panels correspond to the LUMO, and the lower panels to the HOMO. The four states have $E_{1}$ symmetry. Red and blue indicate positive and negative $( \pm 0.01$ a.u. $)$ values of the wave function.

the TB results and the DFT results for the finite nanotube, we conclude that the DOS of the finite tube with border effects excluded reproduces the main characteristics of the DOS of the semiconducting infinite nanotube, including the gap, as is also the case for finite elongated graphene nanoribbons. ${ }^{11}$

Magnetic effects are expected in the system due to the large state degeneracy at the Fermi level. ${ }^{9,33,34}$ The DOS obtained when the electronic spin degree of freedom is included in the calculation is given in Fig. 6. This corresponds to the actual ground state of the system, a state with $S=0$ and antiferromagnetic order between the spins of the electrons in the states localized at the opposite edges of the nanotube. A similar result was found for ZGNRs both infinite ${ }^{1,2}$ and finite. ${ }^{3}$ Figure 6(a) shows that the degeneracy at the Fermi level observed in the NM state of Fig. 4(a) (obtained in the locally saturated spin calculation) has been partially removed and, furthermore, an electronic gap shows up both for $\alpha$ - and $\beta$-spin flavors. Again, a comparison of panels a and b of Fig. 6 shows that the HOMO and lowest unoccupied molecular orbital (LUMO) and the states near them are fully localized on the external carbon rings [those edge states have been excluded to plot Fig. 6(b)]. The spin-polarized density of states in the energy region near the HOMO and LUMO states is shown in Fig. 7, where we also present as insets the wave functions for the $A_{1}$ states below the HOMO and above the LUMO.

The spatial distribution of the $E_{1}$ frontier spin orbitals of the $S=0$ AFM ground state indicated in the DOS of Fig. 7 are given in Figs. 8 and 9. Figure 8 shows the HOMO- $\alpha$ and the LUMO- $\alpha$. The HOMO- $\alpha$ (a doubly degenerate $E_{1}$ state) is localized on the left side of the tube, and the LUMO- $\alpha$ (also a doubly degenerate $E_{1}$ state) is localized on the right side. Figure 9 then shows that the HOMO- $\beta$ is localized on the right side, and the LUMO- $\beta$ on the left side of the nanotube. The HOMO- $\alpha$ and HOMO- $\beta$ states are degenerate in energy, as well as the LUMO- $\alpha$ and LUMO- $\beta$ states. The energy gap, calculated as the difference between the energy eigenvalues of the LUMO and HOMO states, is $0.4 \mathrm{eV}$ for both spin channels. Just below the HOMO- $\alpha$ and HOMO- $\beta$, $60 \mathrm{meV}$ more bound, there are two other states, one $\alpha$ and another $\beta$ both with $A_{1}$ symmetry, also well localized at the

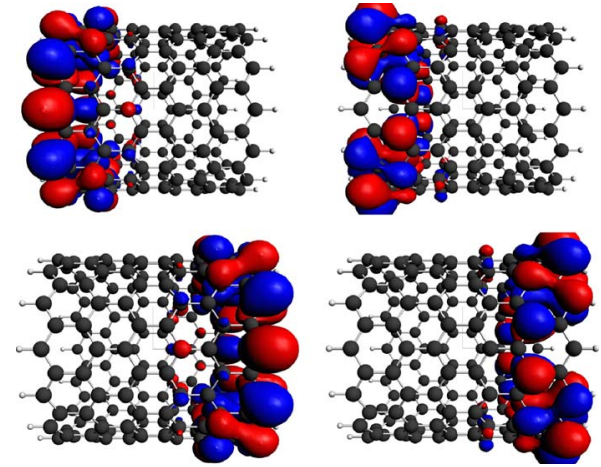

FIG. 9. (Color online) Like Fig. 8 but now for $\beta$ spin. The upper panels represent the LUMO, and the lower panels the HOMO. The four orbitals have $E_{1}$ symmetry.

corresponding edges of the nanotube: $A_{1} \alpha$ on the left side and $A_{1} \beta$ on the right side. There are also two degenerate unoccupied states, again one $\alpha$ and another $\beta$, both of $A_{1}$ symmetry, nearly degenerate in energy with the LUMO- $\alpha$ and LUMO- $\beta$ (only $20 \mathrm{meV}$ less bound) which are also well localized at the edges: $A_{1} \alpha$ on the right side and $A_{1} \beta$ on the left side. So, the collection of edge states for these finite nanotubes includes one $A_{1}$ and two $E_{1}$ occupied states and again two $E_{1}$ and one $A_{1}$ unoccupied states for the two spin flavors $\alpha$ and $\beta$; the evolution of these edge states due to the presence of an external electric field is discussed in Sec. III C. All the other molecular orbitals, those in Fig. 6(b), are delocalized over the whole nanotube with wave functions analogous to those included in the insets of Fig. 4(b). The net spin density $\rho_{\alpha}(\vec{r})-\rho_{\beta}(\vec{r})$ is shown in Fig. 10 to remark the spin imbalance at the nanotube edges due to the localized edge states. A further analysis of the spin properties of the $S=0$ AFM ground state in the absence of electric field is displayed in Fig. 11 where we present the spin $\alpha$ and $\beta$

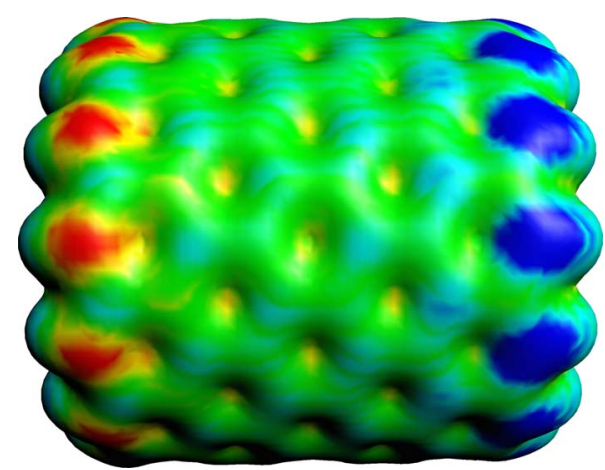

FIG. 10. (Color online) Net spin density $\rho_{\alpha}(\vec{r})-\rho_{\beta}(\vec{r})$ for $\mathrm{C}_{168} \mathrm{H}_{28}$. The net spin density (in a.u.) is indicated by the different colors: 0.0003 , red; 0.00015 , yellow; 0.00 , green; -0.00015 , light blue; and -0.00030 , dark blue. The left side of the tube is dominated by $\alpha$-spin density (the red color indicates that $\alpha$-spin density is greater than $\beta$-spin density on the left), and the right side is dominated by $\beta$-spin density (the blue color shows that $\beta$-spin density is greater than $\alpha$-spin density). The largest differences between $\alpha$ - and $\beta$-spin densities occur over the carbon atoms at the edges of the nanotube. (The surface used to project the colored net spin values corresponds to $\alpha$-spin density of 0.0010 a.u.) 

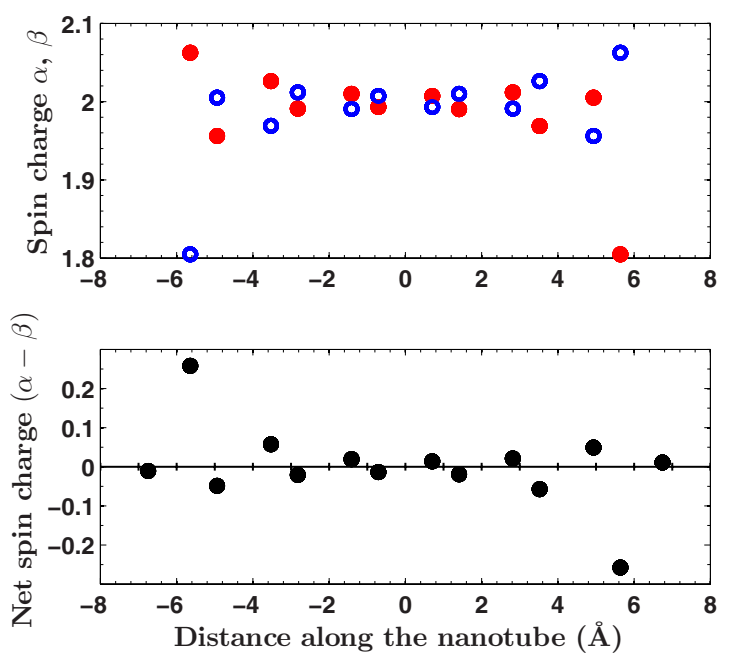

FIG. 11. (Color online) Mulliken $\alpha$ - and $\beta$-spin charges per atom along the nanotube for the AFM ground state $(S=0)$ at zero electric field. In the upper panel, red filled dots represent $\alpha$-spin populations and empty blue dots $\beta$ spin. The spin populations are plotted for the carbon atoms but not for the hydrogen atoms at the boundaries. The lower panel gives the difference between $\alpha$ and $\beta$ populations. The majority spin is $\alpha$ on the leftmost carbon ring and $\beta$ on the rightmost carbon ring, thus showing the AFM configurations of the state. The two values at $\pm 6.745 \AA$ correspond to the hydrogen rings.

Mulliken atomic populations and their difference for the different locations of the carbon atoms along the finite nanotube. The sum of $\alpha$ and $\beta$ charges gives 4 , the number of valence electrons of each carbon atom. From the lower part of Fig. 11, we obtain that the maximum value of the Mulliken spin polarization of the zigzag edge carbon atoms is 0.26 , in agreement with the typical LSDA results quoted in Ref. 3 for finite nanoribbons. The antiferromagnetic structure of the state is clearly displayed in Figs. 10 and 11. Furthermore, the data indicate a spin charge oscillation which is damped as it progresses inside the nanotube.

\section{Half-metallicity induced by the electric field}

In order to study the onset of half-metallicity in the finite nanotube under the influence of an external axial electric field, we have analyzed the behavior of the $\alpha$ and $\beta$ band gaps for the collection of $S=0$ AFM ground states corresponding to different values of the electric field. The binding energies of these states are those at $\left(n_{\alpha}-n_{\beta}\right)=0$ in Figs. 1 and 2. The electrostrictive response of carbon nanotubes to an external electric field modifies their length. ${ }^{35}$ According to DFT calculations, ${ }^{36}$ an electric field of $0.1 \mathrm{~V} / \AA$ induces elongations of $2.9 \%$ on clean finite nanotubes. However, the effect is much reduced on hydrogen terminated nanotubes. ${ }^{36}$ Based on the last result, in the present calculations the atomic structure of the hydrogenated finite nanotube has been fixed to the minimum-energy geometry obtained at zero electric field. So we describe in the following the electronic relaxation with a fixed geometrical structure. For a finite system, the band gaps correspond to the LUMO-HOMO energy difference. The band gaps as a function of the intensity of the

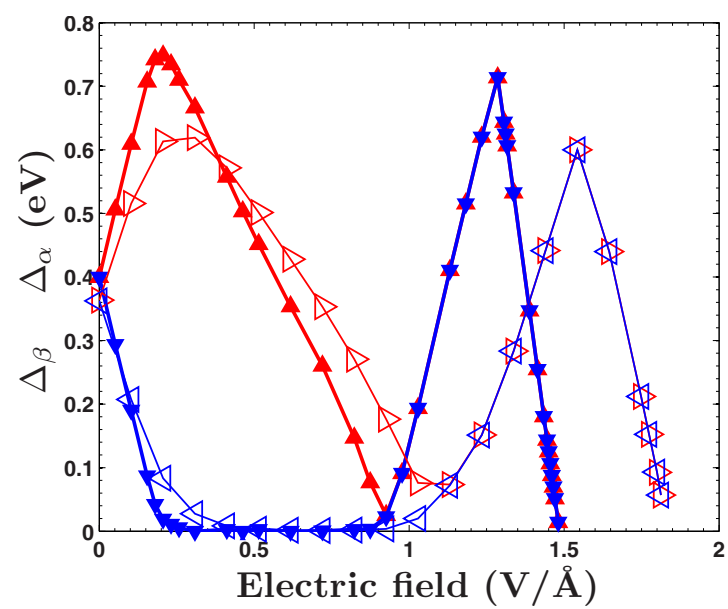

FIG. 12. (Color online) Evolution of $\Delta_{\alpha}$ and $\Delta_{\beta}$, the $\alpha$ (red) and $\beta$ (blue) electronic gaps, as a function of the intensity of the electric field. As the field grows until $E=0.2 \mathrm{~V} / \AA$, the $\alpha$ gaps increase and the $\beta$ gaps decrease. Full and empty symbols are for $\mathrm{C}_{168} \mathrm{H}_{28}$ and $\mathrm{C}_{112} \mathrm{H}_{28}$ tubes, respectively. In all cases the gaps correspond to the ground state with $S=0$.

electric field are given in Fig. 12. The evolution is analogous to that described for nanoribbons $s^{1,2}$ and for nanoislands. ${ }^{3}$ We have included in Fig. 12 the results for the short and the long nanotubes, but in the following we discuss mainly the results for the long nanotube, $\mathrm{C}_{168} \mathrm{H}_{28}$. One can observe in Fig. 12 that in the absence of an electric field, the $\alpha$ and $\beta$ gaps are equal. Then, as the field increases, the $\alpha$ gap increases and the $\beta$ gap decreases. The system becomes half-metallic around $E=0.20 \mathrm{~V} / \AA$ when the $\beta$ gap is actually zero, and the $\alpha$ gap reaches its maximum value. This value of the critical field is nearly identical to that obtained in Ref. 2 for nanoribbons. In Fig. 13 we present the relative variation of the $\alpha$ and $\beta$ gaps for both finite nanotubes in terms of the potential difference between the two extremes of the nanotube. As in the case of ZGNRs, ${ }^{1}$ the critical field to establish half-metallicity in finite zigzag SWNTs is estimated to be $3.0 / w(\mathrm{~V} / \AA)$, where $w$ is the length of the nanotube.

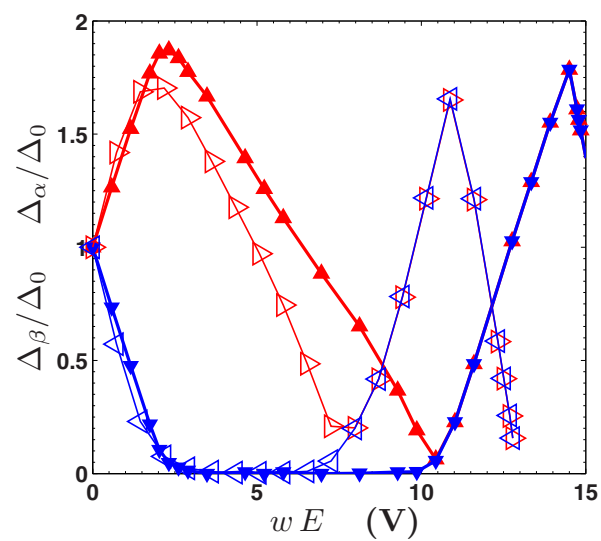

FIG. 13. (Color online) Normalized values of the electronic gaps of Fig. 12, $\Delta_{\alpha}(E) / \Delta_{\alpha}(0)$ (red) and $\Delta_{\beta}(E) / \Delta_{\beta}(0)$ (blue), where $\Delta_{\alpha}(0)=\Delta_{\beta}(0)=\Delta_{0}$, in terms of the potential difference $w E$ between the extremes of the nanotube. Full and empty symbols like in Fig. 12 . 


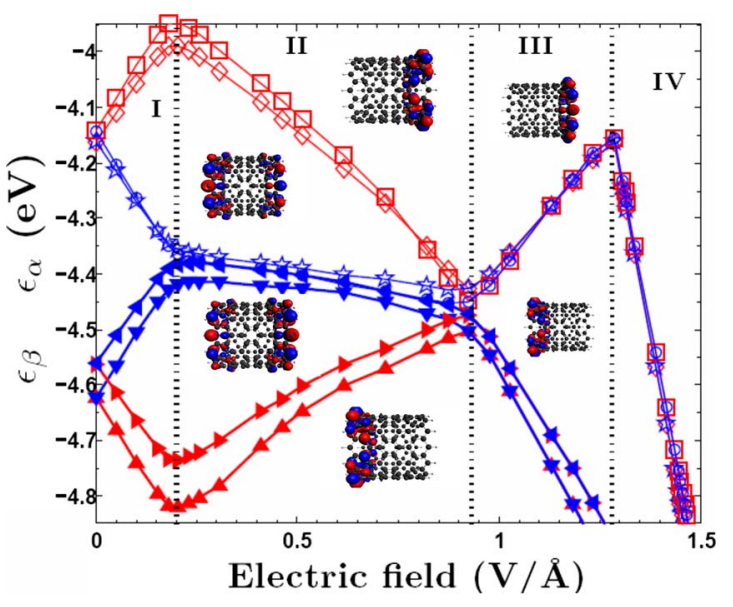

FIG. 14. (Color online) Energy eigenvalues $\epsilon_{\alpha}$ and $\epsilon_{\beta}$ of the edge states of $\mathrm{C}_{168} \mathrm{H}_{28}$ as a function of the intensity of the electric field. The electric field is directed from left to right. Full symbols indicate occupied states, and empty symbols unoccupied states. Lines are red for $\alpha$-spin and blue for $\beta$-spin states. The different symbols indicate, from the top to the bottom in region I, (a) (unoccupied states, empty symbols) square: $A_{1} \alpha$, diamond: $E_{1} \alpha$, circle: $A_{1} \beta$, and five point star: $E_{1} \beta$ and (b) (occupied states, filled symbols) triangle pointing to the left: $E_{1} \beta$, triangle pointing down: $A_{1} \beta$, triangle pointing to the right: $E_{1} \alpha$, and vertical triangle: $A_{1} \alpha$. The insets correspond to the wave functions of some orbitals and they are explained in the text.

Continuing with the description of the results for the long tube, Fig. 12 indicates that in the region of $0.2-0.9 \mathrm{~V} / \AA$, the system maintains the half-metallic behavior with a decreasing value of the $\alpha$ gap. This region of half-metallicity, about $0.7 \mathrm{~V} / \AA$ wide, is slightly wider than the maximum one found in Ref. 2 for fully hydroxylated ZGNRs. Finally, for $E=0.9 \mathrm{~V} / \AA$ the nanotube reaches the region in which the $\alpha$ and $\beta$ gaps are again identical, a result also obtained for oxidized ZGNRs. ${ }^{2}$ For stronger electric fields, the two gaps increase and a new maximum is found at $E$ $=1.29 \mathrm{~V} / \AA$ for the, now equal, $\alpha$ and $\beta$ gaps. Finally, full metallic behavior for both spins is predicted for more intense electric fields at $E=1.5 \mathrm{~V} / \AA$. The variation of the spin gaps just described is due to the behavior of the localized edge states when the electric field is increased. This behavior is given in Fig. 14, where four different regions are considered in correspondence with the behavior of the gaps in Fig. 12. In region I, the edge states close to the HOMO and LUMO are spatially localized, as shown in Figs. 8 and 9. So, as the electric field increases (the field is directed along the nanotube from left to right) the energies of the states localized on the left-hand side (HOMO- $\alpha$ and LUMO- $\beta$ ) become more negative (the binding energy of those states increases) and the binding energy of the states localized on the right side (LUMO- $\alpha$ and HOMO- $\beta$ ) decreases. This increases the $\alpha$ gap and decreases the $\beta$ gap. In region II, from the critical field $E=0.2 \mathrm{~V} / \AA$ until $E=0.9 \mathrm{~V} / \AA$, the $\beta$ states near the Fermi level, both occupied and empty, are much less affected by the electric field due to their spatial localization on both sides of the nanotube, as is clear from the two insets at the left of region II showing the wave functions corresponding to

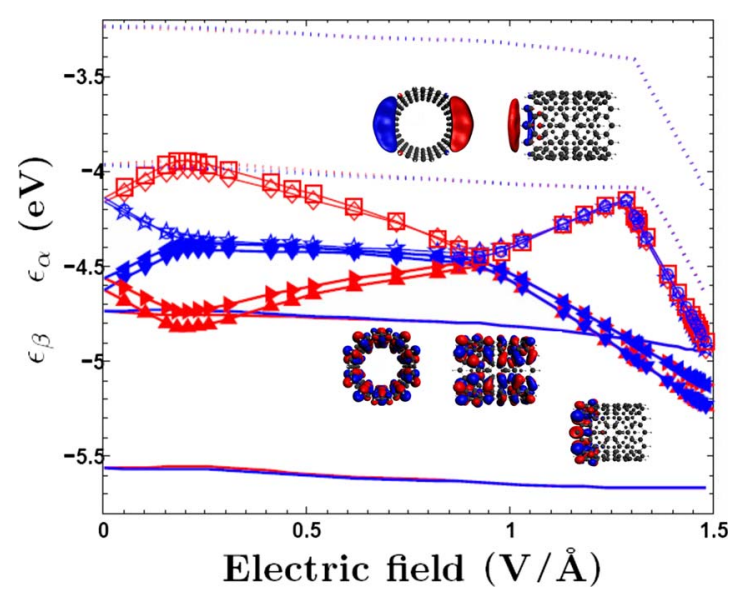

FIG. 15. (Color online) Electric field dependence of the energy eigenvalues of the molecular orbitals around the Fermi level for $\mathrm{C}_{168} \mathrm{H}_{28}$. Comparing with Fig. 14, the new continuous lines correspond to occupied $E_{3}$ (at $\left.-5.5 \mathrm{eV}\right)$ and $E_{2}$ states, and the dashed lines to empty states of $E_{3}$ (the less bound states) and $E_{2}$ symmetry. These states where indicated in Fig. 6(b) for $E=0$. The insets correspond, from top to bottom, to two views of the LUMO, two views of the HOMO, and one view of the first state below the HOMO; all views for an electric field $E=1.34 \mathrm{~V} / \AA$.

the HOMO- $\beta$ and LUMO- $\beta$ at an intermediate value of the field, $E=0.5 \mathrm{~V} / \AA$. For the same field, the other two insets in region II show that the HOMO- $\alpha$ and LUMO $-\alpha$ orbitals are still localized on opposite edges but these now have a sizable contribution of the second, more internal, chain of carbon atoms. So, for values of the electric field in region II, an increasing electric field produces this partial delocalization toward the internal part of the nanotube and the corresponding change in energy of the eigenvalues. In region III, beginning around $E=0.9 \mathrm{~V} / \AA$, a new reorganization of the frontier orbitals occurs, and the occupied $\alpha$ and $\beta$ orbitals become localized on the left side of the tube, while empty states near the LUMO are localized on the right side; this is shown in the two insets for the HOMO and LUMO states corresponding to a field $E=0.93 \mathrm{~V} / \AA$. For electric fields in region III, the $\alpha$ and $\beta$ states are degenerate in energy and their wave functions are identical for a common state symmetry. In this region the electric field is strong enough to induce a displacement of the charge located in the orbitals near the HOMO states; this charge is displaced into regions of lower total potential energy. Finally, in region IV, beginning at $E=1.3 \mathrm{~V} / \AA$, the LUMO orbitals are localized on the left side of the nanotube due to the strong increase in the electrostatic potential produced by the electric field in that region, and consequently their energies become more negative as the electric field increases. The evolution of the electronic states near the HOMO is given in Fig. 15, where we show not only the energy eigenvalues of the edge states like in Fig. 14 but also the energy eigenvalues of the delocalized states that are closer in energy. The continuous lines starting at -5.6 and $-4.7 \mathrm{eV}$ correspond to occupied orbitals with symmetries $E_{3}$ and $E_{2}$, respectively, and the dotted lines starting at -4.0 and $-3.24 \mathrm{eV}$ correspond to empty orbitals with $E_{2}$ and $E_{3}$ symmetries. The energies of these delocalized states have a very small dependence on the electric field 
for the range of fields corresponding to regions I, II, and III of Fig. 14. However, starting at $E=1.3 \mathrm{~V} / \AA$ and due to the level crossing displayed, the actual HOMO state of the system is the $E_{2}$ delocalized state; the delocalization of the orbital is clear in the lateral and front views given in the inset. The wave function for the first degenerate state under the HOMO, which is still an edge state, is also shown. At these strong fields the degenerate LUMO orbitals become localized with most of their charge outside the edge carbon atoms (see lateral and front views in the upper inset of Fig. 15). Of course, these states are strongly influenced by the electric field, thus producing the rapid linear decrease in the HOMOLUMO gap shown in Fig. 12. We remark that these states near the LUMO are empty states, and only at the largest electric field of $E=1.5 \mathrm{~V} / \AA$, when the gap is closed again for both spins, the system experiences field ionization when one of those orbitals localized outside the nanotube becomes occupied by one electron. This field can be considered as the threshold for the onset of field emission from the finite hydrogenated nanotube.

\section{CONCLUSIONS}

DFT calculations using the LSDA for exchange and correlation predict that finite zigzag $(14,0)$ SWNTs with hydrogenated edges present half-metallic behavior induced by an external electric field applied parallel to the axis of the nanotube. This result is analogous to the behavior described for edge passivated zigzag carbon nanoribbons ${ }^{1,2}$ and also for finite rectangular nanoislands with zigzag edges. ${ }^{3}$ Furthermore, the critical electric field required to establish halfmetallicity is around $3.0 / w(\mathrm{~V} / \AA)$, where $w$ is the length of the nanotube, in full agreement with the value obtained for ZGNRs of width $w .{ }^{1}$ Half-metallicity results from the peculiar electronic structure of the ground state of the finite nanotube in the absence of electric field. The HOMO and LUMO, and those orbitals very close to these, have the character of edge states: these are spatially localized on the carbon atoms at the ends of the tube. Moreover, the ground state is an antiferromagnetic state in which one end of the nanotube is dominated by $\operatorname{spin} \alpha$ states and the other side by spin $\beta$ states, coupled to a total spin $S=0$. The electric field has a larger influence on these localized edge states than on the rest of the molecular orbitals, which are delocalized along the nanotube, thus producing the decrease in the electronic gap for one spin flavor and the increase in the gap of the other spin. We have also analyzed the magnetic properties of these finite nanotubes. For all the electric-field intensities considered, the ground state has the AFM structure described above, and there is a large energy difference between this AFM state and the NM state of 577 and $480 \mathrm{meV}$, respectively, for the long and short finite nanotubes studied. In the NM state, also with $S=0$, the edge states are equally populated by $\alpha$ - and $\beta$-spin electrons. However, at least for electric fields $E$ between 0 and $0.51 \mathrm{~V} / \AA$ there is a second local energy minimum with net spin $S$ different from zero. For field values $E<0.2 \mathrm{~V} / \AA$ we obtain $S=3$, and for $E$ $>0.4 \mathrm{~V} / \AA$ we obtain $S=5 / 2$ and $S=2$ as the field increases. The energy difference between these local minima and the corresponding $S=0$ AFM ground state is only $45 \mathrm{meV}$ for $\mathrm{C}_{168} \mathrm{H}_{28}$ and $50 \mathrm{meV}$ for the shorter tube $\mathrm{C}_{112} \mathrm{H}_{28}$. These energy differences are in agreement with those recently reported for finite nanoribbons. ${ }^{3}$ However, thermal transitions between both states are not expected because the calculated energy barriers are in the range of 100-300 meV. These energy barriers can be considered as upper bounds, as far as noncollinear spin structures can lower their actual values. The large energy differences between the NM and the FM states (the FM states are more stable by 3.43 and $4.3 \mathrm{meV}$ per carbon atom for the long and short tubes) indicate the possibility of implementing stable nanomagnets using finite pieces of zigzag carbon nanotubes.

We have also calculated the longitudinal dipole polarizabilities, which show finite-size effects and are substantially smaller than the corresponding polarizabilities for infinite SWNTs, but of the same order of magnitude as those corresponding to typical finite carbon nanosystems such as fullerens. Preliminary results for finite zigzag $(7,0)$ SWNTs indicate the same qualitative behavior as described for the two finite $(14,0)$ SWNTs, including the same value for the critical electric field required to establish half-metallicity. Finally, we think that our results are of relevance in the implementation of nanodevices because nanotubes of finite length should be used in those devices. Furthermore, the halfmetallic behavior found at easily accessible polarization potentials makes finite nanotubes relevant systems in spintronics.

\section{ACKNOWLEDGMENTS}

Research supported by MEC of Spain (Projects No. MAT2005-06544-C03-01, No. MAT2005-06544-C03-03, No. FIS2004-06490-C03-00, and MONACEM), by Junta de Castilla y León (Project No. VA039A05), by Basque Government (Project No. IE05-151) under the ETORTEK Program (NANOMAT), and by the European Network of Excellence NANOQUANTA (Grant No. NM4-CT-2004-500198).

\footnotetext{
*angel.mananes@unican.es

${ }^{\dagger}$ Also at Donostia International Physics Center (DIPC), E-20018 San Sebastián, Spain.

${ }^{1}$ Y.-W. Son, M. L. Cohen, and S. G. Louie, Nature (London) 444, 347 (2006); 446, 342 (2007).

${ }^{2}$ O. Hod, V. Barone, J. E. Peralta, and G. E. Scuseria, Nano Lett.
} 
${ }^{7}$ S. A. Wolf, D. D. Awschalom, R. A. Buhrman, J. M. Daughton, S. von Molnár, M. L. Roukes, A. Y. Chtchelkanova, and D. M. Treger, Science 294, 1488 (2001).

${ }^{8}$ L. Pisani, J. A. Chan, B. Montanari, and N. M. Harrison, Phys. Rev. B 75, 064418 (2007).

${ }^{9}$ H. Lee, Y. Son, N. Park, S. Han, and J. Yu, Phys. Rev. B 72, 174431 (2005).

${ }^{10}$ Z. Zhou, M. Steigerwald, M. Hybertsen, L. Brus, and R. A. Friesner, J. Am. Chem. Soc. 126, 3597 (2004).

${ }^{11}$ O. Hod, J. E. Peralta, and G. E. Scuseria, Phys. Rev. B 76, 233401 (2007).

${ }^{12}$ D. Jiang, B. G. Sumpter, and S. Dai, J. Chem. Phys. 127, 124703 (2007).

${ }^{13}$ J. Fernández-Rossier and J. J. Palacios, Phys. Rev. Lett. 99, 177204 (2007).

${ }^{14}$ M. Ezawa, Phys. Rev. B 76, 245415 (2007).

${ }^{15}$ Y. Kobayashi, K. I. Fukui, T. Enoki, K. Kusakabe, and Y. Kaburagi, Phys. Rev. B 71, 193406 (2005).

${ }^{16}$ Y. Niimi, T. Matsui, H. Kambara, K. Tagami, M. Tsukada, and H. Fukuyama, Phys. Rev. B 73, 085421 (2006).

${ }^{17}$ Carbon Nanotubes. Synthesis, Structure, Properties, and Applications, edited by M. S. Dresselhaus, G. Dresselhaus, and Ph. Avouris (Springer-Verlag, Berlin, 2001).

${ }^{18}$ R. Saito, G. Dresselhaus, and M. Dresselhaus, Physical Properties of Carbon Nanotubes (Imperial College, London, 1998).

${ }^{19}$ S. Okada and A. Oshiyama, J. Phys. Soc. Jpn. 72, 1510 (2003).

${ }^{20}$ Y. Higuchi, K. Kusakabe, N. Suzuki, S. Tsuneyuki, J. Yamauchi, K. Akagi, and Y. Yoshimoto, J. Phys.: Condens. Matter 16, S5689 (2004).

${ }^{21}$ Y.-H. Kim, J. Choi, K. J. Chang, and D. Tománek, Phys. Rev. B
68, 125420 (2003).

${ }^{22}$ N. Park, M. Yoon, S. Berber, J. Ihm, E. Osawa, and D. Tománek, Phys. Rev. Lett. 91, 237204 (2003).

${ }^{23}$ G. te Velde, F. M. Bickelhaupt, S. J. A. van Gisbergen, C. F. Guerra, E. J. Baerends, J. G. Snijders, and T. Ziegler, J. Comput. Chem. 22, 931 (2001).

${ }^{24}$ C. Fonseca-Guerra, J. G. Snijders, G. te Velde, and E. J. Baerends, Theor. Chem. Acc. 99, 391 (1998).

${ }^{25}$ S. Vosko, L. Wilk, and M. Nusair, Can. J. Phys. 58, 1200 (1983).

${ }^{26}$ P. Lang, L. Nordström, R. Zeller, and P. H. Dederichs, Phys. Rev. Lett. 71, 1927 (1993)

${ }^{27}$ J. Enkovaara, A. Ayuela, and R. M. Nieminen, Phys. Rev. B 62, 16018 (2000).

${ }^{28}$ G. M. Pastor, J. Dorantes-Dávila, and K. H. Bennemann, Phys. Rev. B 40, 7642 (1989).

${ }^{29}$ G. D. Mahan and K. R. Subbaswamy, Local Density Theory of Polarizability (Plenum, New York, 1990).

${ }^{30}$ L. X. Benedict, S. G. Louie, and M. L. Cohen, Phys. Rev. B 52, 8541 (1995).

${ }^{31}$ S. L. Ren et al., Appl. Phys. Lett. 59, 2678 (1991).

${ }^{32}$ J. W. Ding, X. H. Yan, and J. X. Cao, Phys. Rev. B 66, 073401 (2002).

${ }^{33}$ M. Fujita, K. Wakabayashi, K. Nakada, and K. Kusakabe, J. Phys. Soc. Jpn. 65, 1920 (1996).

${ }^{34}$ S. Okada and A. Oshiyama, Phys. Rev. Lett. 87, 146803 (2001).

${ }^{35}$ W. Guo and Y. Guo, Phys. Rev. Lett. 91, 115501 (2003).

${ }^{36}$ I. Cabria, C. Amovilli, M. J. López, N. H. March, and J. A. Alonso, Phys. Rev. A 74, 063201 (2006). 